\title{
Adding Value to Health Care: Where Radiologists May Contribute
}

\author{
R.A. Charalel, K.D. Hentel, R.J. Min, and P.C. Sanelli
}

$\mathrm{N}^{2}$ ational health care reform and scrutiny on rising health care costs have created a climate in which health care providers must improve the value, or quality per cost, ${ }^{1}$ of patient care. Radiology in particular is at an important crossroads given the heavy use of imaging and the associated large percentage of health care expenditures. $^{2}$ At this critical juncture, it is important for radiologists to take responsibility for delivering the highest possible value of imaging and actively contribute to institutional and national solutions for providing the highest valued patient care. ${ }^{3,4}$ Only with such active participation will radiologists be able to illustrate our role as an important cornerstone for health care delivery and secure our positions as decisive members of the health care team.

\section{HOW RADIOLOGISTS CAN ADD VALUE}

Value is a measure of quality efficiency and has been defined as quality per cost. ${ }^{1}$ Quality in radiology may be defined as the ability to obtain the right imaging examination at the right time with accurate, readily available, and actionable interpretations. There are several steps of the imaging process at which radiologists have the opportunity to further improve patient care and health care efficiency, and serve as valued resources to patients and referring clinicians. ${ }^{5}$ The following are examples of how radiologists can add value in their practice.

\section{ACQUIRING QUALITY IMAGES}

Most imaging orders are created by a referring physician who does not have imaging expertise. Thus, it is not surprising that the initial order may not always be the safest and most effective examination. As imaging experts, radiologists can insure that the right study gets done at the right time in the most effective way (ie, with or without contrast). This "consultation" occurs daily in many practices and is referred to as protocoling. For example, neuroradiologists add considerable value in developing and choosing between complex MR

Received May 7, 2014; accepted after revision June 22.

From the Department of Radiology, New York-Presbyterian Hospital/Weill Cornell Medical Center, New York, New York.

Please address correspondence to Pina C. Sanelli, MD, 1305 York Ave, 3rd Floor, New York, NY 10021; e-mail: pcs9001@med.cornell.edu

http://dx.doi.org/10.3174/ajnr.A4068 imaging protocols to answer a targeted clinical question. These protocols differ when evaluating pathology in different central nervous system locations such as is seen with imaging neoplasms in the brain, skull base, spine, or head and neck. Despite the value of this service, most patients and referring physicians are not even aware of the radiologist's effort. This can be taken a step further by developing radiology consultation services, ${ }^{6}$ thereby formalizing our role as specialist consultants, and facilitating access for both physicians and patients to the radiologist.

As imaging experts, radiologists also serve important institutional roles by contributing to equipment selection, technologist training, and the maintenance of quality metrics to help ensure that diagnostic quality images are produced. Furthermore, radiologists are responsible for developing new image protocols to reduce radiation dose, while still maintaining diagnostic quality. In addition, radiologists monitor radiation dosimetry for all patients, staff, and civilians, enforcing policies to reduce unnecessary exposure. As image experts, radiologists are critical to all aspects of developing a quality image.

\section{IMAGE INTERPRETATION}

Fee-for-service payment models have resulted in radiologists being rewarded for the efficient and accurate interpretation of imaging studies. However, value should not be measured on the basis of a single imaging study but rather considered in the context of the patient's medical condition. By using tools and information increasingly available to the radiologist at the time of image interpretation, the radiologist may create reports that not only describe the imaging study performed but increase value by guiding the care of the imaged patient. Radiologists must take into context the clinical scenario and put forth interpretations that are relevant with differential diagnoses that are plausible. The increasing availability of electronic medical records allows the radiologist to review images with the added context provided by operative notes, outpatient clinic notes, laboratory testing, and pathology results. Such context often results in a more informed and useful differential diagnosis; for example, identifying focal neurologic symptoms helps to focus the image interpretation of patients with acute stroke and may lead to the increased discovery of subtle findings such as a hyperattenuated vessel sign or early ischemic changes that may otherwise be overlooked. When ade- 
quate information is not readily available in the medical record, consultations with the ordering physicians, other members of a patient's health care team, and the patient serve to acquire the necessary information and increase the visibility of the radiologist.

Furthermore, we can enhance the relevance of our interpretations by providing reports that synthesize all available imaging modalities across all organ systems, not just the most recent prior examination of a specific technique or body part. Subspecialization in radiology has led to nuanced reporting from expert imagers, particularly in the case of neuroradiology studies, which are largely interpreted by subspecialists in academic institutions and even in the community. However, it can also result in multiple reports for a single patient, even within the same care episode, leaving referring physicians to reconcile these multiple and potentially conflicting reports even though they may not have the expertise to understand the nuanced reports and the strengths and limitations of the used imaging modalities, or the ability to re-examine the images in a holistic patient context. Radiologists as imaging experts have the skills to do so, uniquely qualifying them to improve service and optimize care for our patients.

The greatest opportunity to provide value is when patients are new to a health care system and arrive with prior outside imaging. By facilitating the availability of such imaging to the new health care system and providing reports summarizing findings in a holistic patient context, repeat and often unnecessary studies can be reduced, ultimately allowing for more efficient and effective care of the patient.

\section{BEYOND IMAGE INTERPRETATION}

Still, providing more valuable reports is not enough. The value provided by the radiologist must be demonstrated to patients and the medical community. Failure to do so will risk the commoditization of imaging and places radiology in an unfavorable position as health care continues to transition to accountable, bundled care. Several opportunities for increased recognition exist in many of our practices. Multidisciplinary care conferences, including tumor boards and rounds, serve as an opportunity for radiologists to demonstrate their value within the overall care team by providing an opportunity to synthesize a patient's imaging history and suggest additional imaging studies as needed.

In addition, maintaining and developing technology for direct access to radiology reports and images through patient portals provides increased exposure. However, currently, most radiologists do not create reports with the patient audience in mind. Moving forward, radiologists and their reports must be cognizant of this new audience. ${ }^{7}$ Finally, achieving the visibility needed will no doubt require radiologists to leave the reading room and directly interact with patients. In the new paradigm of decentralized reading rooms within clinics, patients and referring physicians may be more accessible than ever before. ${ }^{8}$

\section{CONCLUSIONS}

Although many clinicians, administrators, and patients may be relatively unaware of the radiologist's valuable role in delivering health care, the services radiologists provide in acquiring quality images, image interpretation, and consultation have been recognized as integral aspects of the physician work component in the Medicare resource-based relative value scale (RBRVS) payment system. Under the RBRVS system, a physician's work is measured by the technical skill, physical effort, required mental effort, judgment, stress due to the potential risk to the patient, and time it takes to perform the service. ${ }^{9}$ However, many opportunities exist for radiologists to improve and demonstrate the value they provide to patients and the health care system. Recent and future changes to the health care environment, including increased emphasis on value and accountable care, provide unprecedented imperative and opportunity to do so.

\section{REFERENCES}

1. Porter ME. What is value in health care? N Engl J Med 2010; 363:2477-81

2. Rao VM, Levin DC. The overuse of diagnostic imaging and the Choosing Wisely initiative. Ann Intern Med 2012;157:574-76

3. Duszak R Jr. Value: imaging's new wave imperative. J Am Coll Radiol 2013;10:484-85

4. American College of Radiology. Imaging 3.0. http://www.acr.org/ Advocacy/Economics-Health-Policy/Imaging-3. Accessed June 10, 2014

5. Patti JA, Berlin JW, Blumberg AL, et al. ACR white paper: the value added that radiologists provide to the health care enterprise. $J \mathrm{Am}$ Coll Radiol 2008;5:1041-53

6. Khorasani R, Silverman SG, Meyer JE, et al. Design and implementation of a new radiology consultation service in a teaching hospital. AJR 1994;163:457-59

7. Kuhlman M, Meyer M, Krupinski EA. Direct reporting of results to patients: the future of radiology? Acad Radiol 2012;19:646-50

8. Rosenkrantz AB, Lepor H, Taneja SS, et al. Adoption of an integrated radiology reading room within a urologic oncology clinic: initial experience in facilitating clinician consultations. J Am Coll Radiol 2014;11:496-500

9. American Medical Association. Overview of the RBRVS. http:// www.ama-assn.org/ama/pub/physician-resources/solutions-managingyour-practice/coding-billing-insurance/medicare/the-resource-basedrelative-value-scale/overview-of-rbrvs.page?. Accessed June 10, 2014 